\title{
Deposited Charge Measurements on Silicon Wafers After Plasma Treatment
}

\author{
J. L. Shohet, K. Nauka, and P. Rissman
}

\begin{abstract}
In plasma processing, especially during the etching process in microelectronics, and as the feature size decreases, charging damage to thin gate oxides can be produced which does not occur when wet chemical processes are used. It is currently believed that such damage occurs when excess charge is deposited on a wafer because of nonuniformities in the plasma parameters across the surface of the wafer. To predict the occurrence of charging damage, unpatterned wafers are exposed to a plasma in which nonuniformity is introduced across the wafer surface. Surface photovoltage (SPV) and contact potential difference (CPD) techniques can be used to determine the regions where excess charge is deposited and thus where the potential for charging damage exists. Wafer maps of these measurements are made to show the difference between uniform and nonuniform charge distributions.
\end{abstract}

$\mathbf{F}$ IOR THE NEXT generation of microelectronics fabrication, in-line monitoring techniques which can detect the potential for plasma-induced damage [1] need to be developed. We present here two methods that can be used in this regard: surface photovoltage (SPV) and contact potential difference (CPD) measurements [2].

An SPV signal is obtained by illuminating one side of a wafer with photons having energies higher than the $\mathrm{Si}$ bandgap, while the other side remains in darkness. Carriers generated near the illuminated surface introduce a charge imbalance resulting for a partial collapse of the surface potential barrier and producing a potential difference across the wafer. At low light intensities, the voltage is directly related to the minority carrier diffusion length [3] and the surface recombination rate. At high light intensities, the surface barrier disappears and the potential difference saturates, and thus it provides information about the magnitude of the surface barrier. The barrier value can be translated into a surface charge or, in the case of an oxidized Si wafer, into the mean value of oxide charge [4]. This means that the measured saturated SPV voltage across an oxidized wafer is proportional to the amount of charge that has been deposited at that point on the wafer.

The CPD measurement technique employs a vibrating Kelvin probe to measure the difference between a reference metal probe and the work function of a measured wafer. In the case of an oxidized Si wafer, the work function of the

Manuscript received August 29, 1995. This work was supported in part by the National Science Foundation under Grant EEC-8721545.

J. L. Shohet is with the Engineering Research Center for Plasma-Aided Manufacturing, University of Wisconsin-Madison, Madison, WI 53706 USA.

K. Nauka and P. Rissman are with the Hewlett-Packard Company, Palo Alto, CA 94304 USA.

Publisher Item Identifier S 0093-3813(96)02181-9.

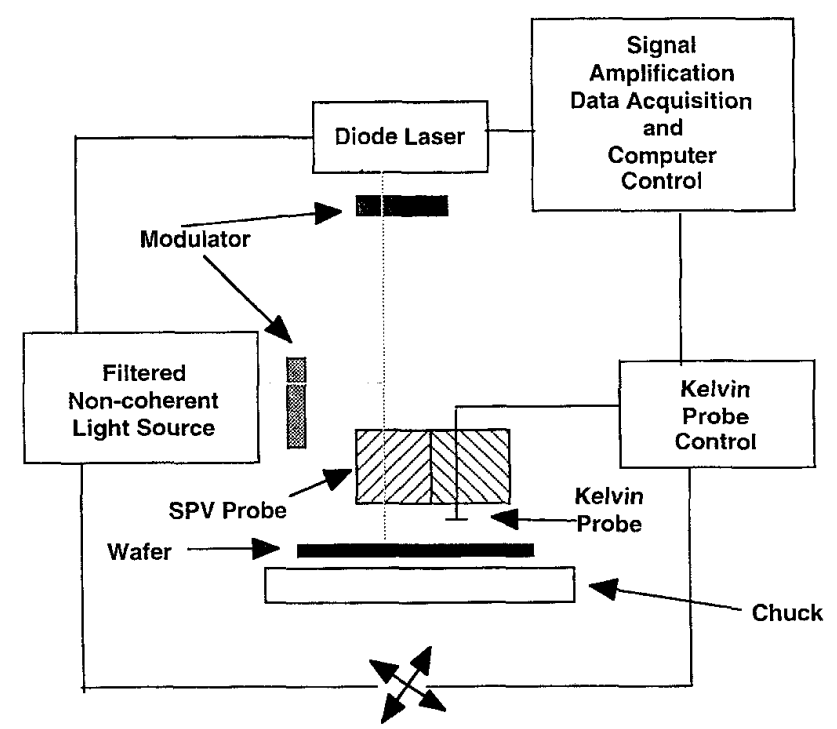

Fig. 1. Experimental setup for the SPV and CPD measurements.

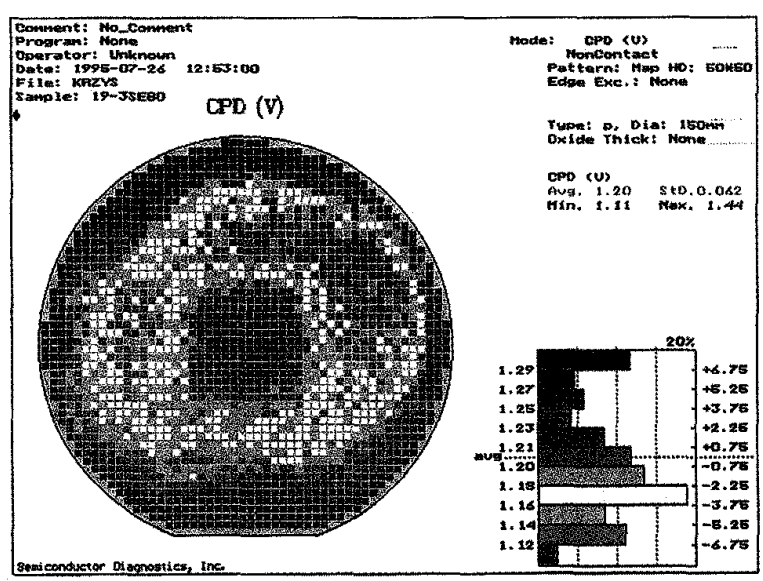

Fig. 2. Measured CPD voltage wafer map

wafer is determined by the oxide surface charge [5]. Thus, when the work function of a Kelvin probe is known, CPD can provide information about the electrical charge stored on the oxide surface. Fig. 1 shows the experimental setup for the SPV and CPD measurements.

The SPV signal was obtained using a filtered noncoherent light source for the minority carrier diffusion length measure- 


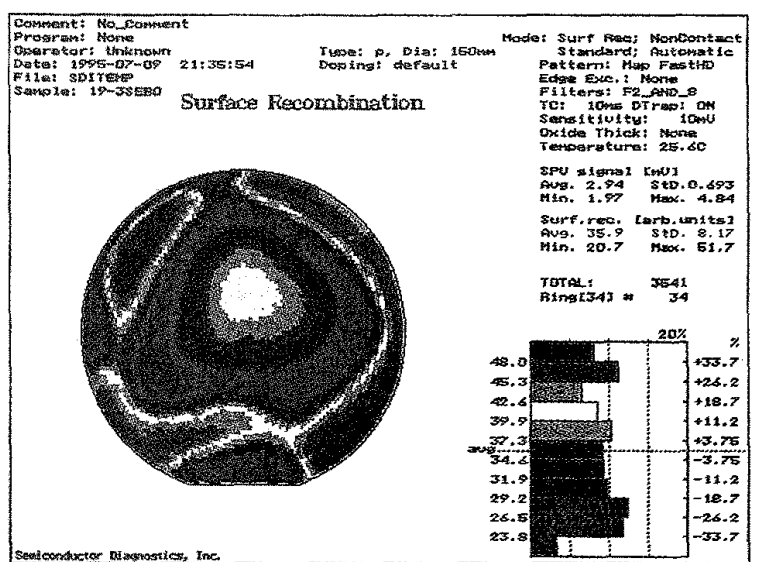

Fig. 3. Measured diffusion length wafer map.

ments, and a diode laser was employed for the surface barrier measurements.

Figs. 2-4 show three representative wafer maps of diffusion length, surface recombination, and CPD voltage for a wafer exposed to a plasma in a parallel plate etcher in which the gap spacing was varied so as to produce nonuniformities in the radial direction. It is clear from the maps that the structure shown by all three wafer maps is quite similar. This implies that both the surface and volume charge densities vary in a similar way.

\section{ACKNOWLEDGMENT}

Special thanks are due to Semiconductor Diagnostics, Incorporated for providing the wafer maps.

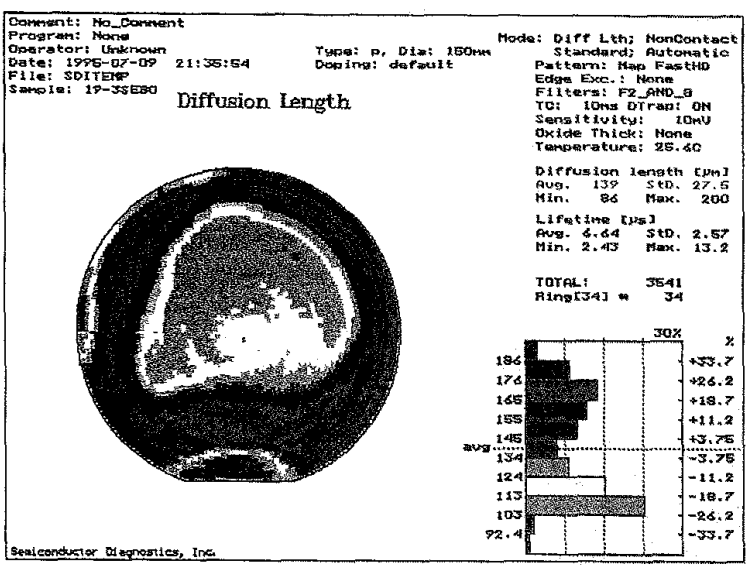

Fig. 4. Measured surface recombination wafer map.

\section{REFERENCES}

[1] S. Fang and J. P. McVittie, "A model and experiments for thin-oxide damage from wafer charging in magnetron plasmas," IEEE Electron Device Lett., vol. 13, p. 347, 1992.

[2] K. Nauka, "Application of surface photovoltage and contact potential difference for in-line monitoring of IC processes," in Proc. Int. Wkshp. Semiconduct. Characterization: Present Status Future Needs, NIST, Gaithersburg, MD, 1995.

[3] J. Lagowski, P. Edelman, M. Dexter, and W. Henley, "Non-contact mapping of heavy metal contamination for silicon IC fabrication," Semiconduct. Sci. Technol, vol. 7, p. A185, 1992.

[4] P. Edelman, J. Lagowski, and L. Lastrzebski, "Surface charge imaging in semiconductor wafers by surface photovoltage," in Proc. Mat. Res. Soc. Mtg., San Francisco, 1992.

[5] W. H. Brattain and J. Bardeen, "Surface properties of germanium," Bell. Syst. Tech. J., vol. 32, p. 1, 1953. 